\title{
Correction to: Hepatitis $C$ virus core protein activates $W n t / \beta$-catenin signaling through multiple regulation of upstream molecules in the SMMC-7721 cell line
}

\author{
Jiao Liu ${ }^{1,2} \cdot$ Zengchan Wang $^{1,2} \cdot$ Jia Tang $^{1,2} \cdot$ Renkuan Tang $^{1,2,3} \cdot$ Xiaoliang Shan $^{1,2} \cdot$ Wenlu Zhang $^{1,2} \cdot$ Qingmei Chen $^{1,2} \cdot$
} Fan Zhou ${ }^{1,2} \cdot \operatorname{Ke~Chen~}^{1,2} \cdot$ Ailong Huang ${ }^{1,2} \cdot$ Ni Tang $^{1,2}$

Published online: 4 November 2021

(c) Springer-Verlag GmbH Austria, part of Springer Nature 2021

\section{Correction to: Arch Virol (2011) 156:1013-1023 https://doi.org/10.1007/s00705-011-0943-x}

It recently came to our attention that the original article contains two errors in Fig. 1A and Fig. 2 occurred during the process of figure assembly. The corrected Fig. 1A and Fig. 2 are shown below.
While these errors do not affect the overall conclusion of our reported work, we sincerely apologize for any inconvenience caused by these unintended errors to the journal and its readers.

The original article can be found online at https://doi.org/10.1007/ s00705-011-0943-x.

Ailong Huang

ahuang1964@yahoo.com.cn

$\triangle$ Ni Tang

nitang809@hotmail.com

1 The Second Affiliated Hospital, Chongqing Medical University, Lin Jiang Road, No. 74, Chongqing 400010, Chongqing, China

2 Key Laboratory of Molecular Biology of Infectious Diseases designated by the Chinese Ministry of Education, Chongqing Medical University, Yixueyuan Road, No.1, Chongqing 400016, Chongqing, China

3 Department of Forensic Medicine, Chongqing Medical University, Chongqing, China 
Corrected Fig. 1A:

A

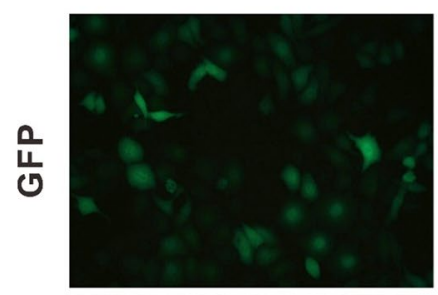

GFP

!

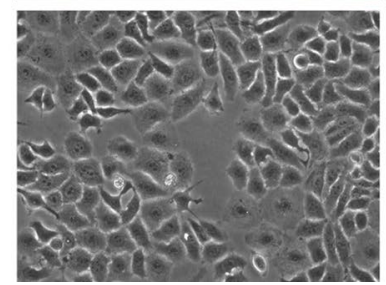

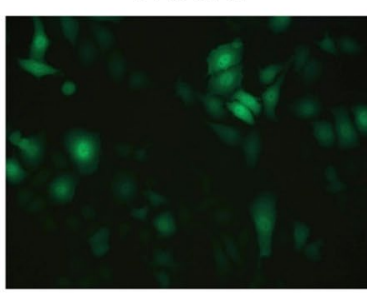

Wnt3a

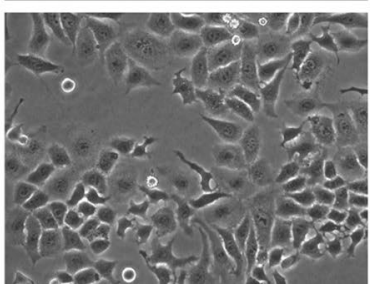

Core
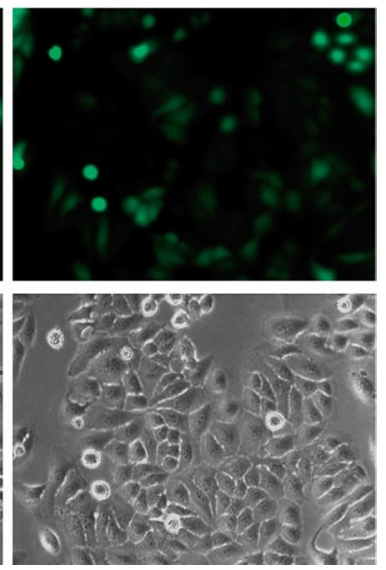

\section{Corrected Fig. 2:}

Fig. 2 Cytosolic accumulation and nuclear translocation of $\beta$-catenin induced by $\mathrm{HCV}$ core. SMMC-7721 cells were infected with AdGFP (panels A to $\mathbf{C}$ ), AdCore (panels $\mathbf{D}$ to F) or AdWnt3A (panels G to I) for $24 \mathrm{~h}$. Cells were fixed and stained with an anti- $\beta$-catenin antibody and a fluorescence labeled secondary antibody. Cells were counterstained with 4', 6-diamidino-2-phenylindole (DAPI) to label nuclei. The presence of $\beta$-catenin was visualized under a confocal immunofluorescence microscope. Nuclear translocation of $\beta$-catenin is indicated by arrows
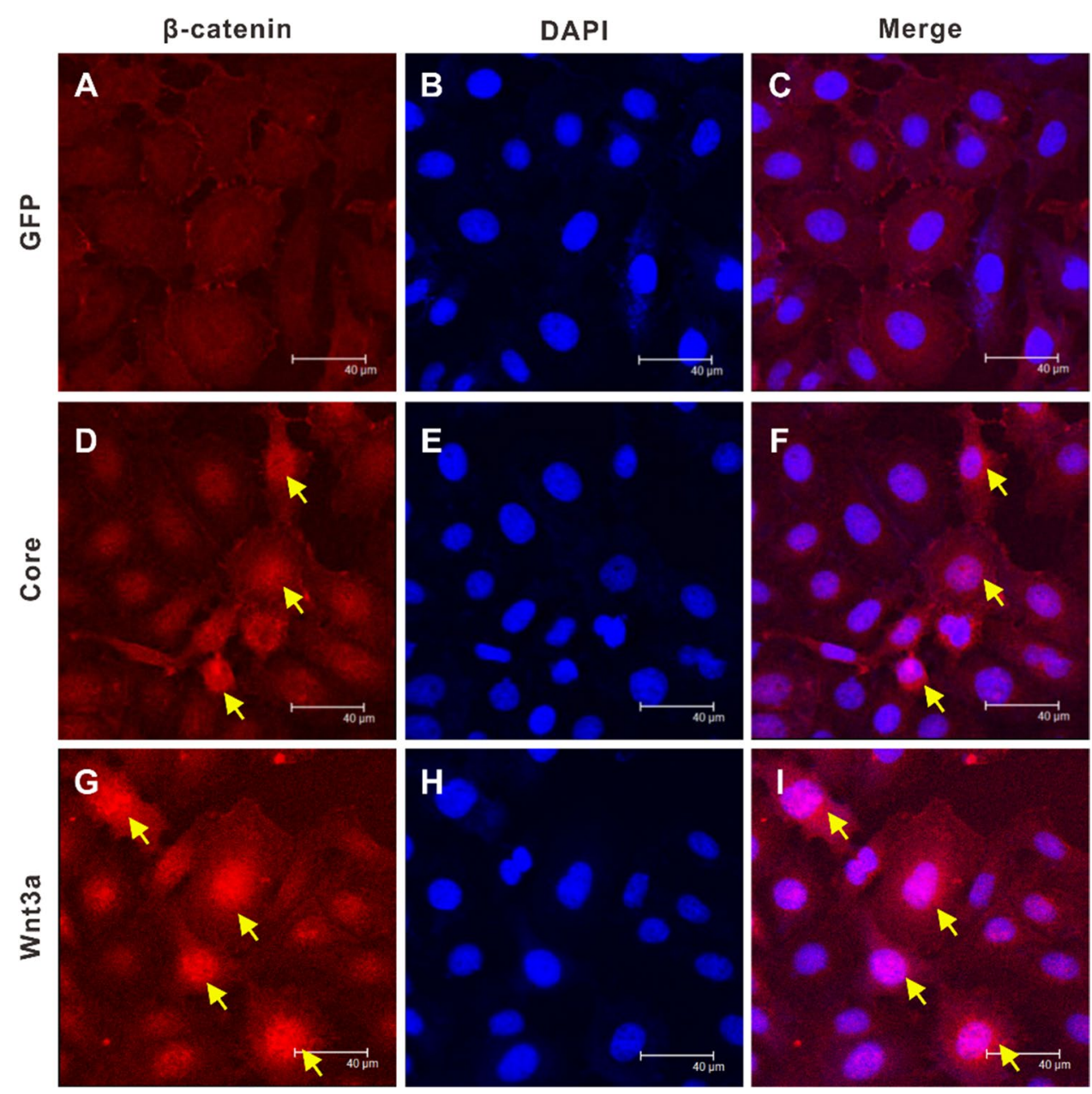

Publisher's Note Springer Nature remains neutral with regard to jurisdictional claims in published maps and institutional affiliations. 